\title{
Spontaneous pneumopericardium in a term newborn: a case report
}

\begin{abstract}
Neonatal pneumopericardium (NPPC) is a rare clinical condition that is usually described in the context of neonatal respiratory diseases that require assisted ventilation. It is usually associated with other air-leak syndromes like pulmonary interstitial emphysema, pneumothorax, pneumomediastinum and subcutaneous emphysema. NPPC can lead to serious complications including sudden cardiac arrest. Prompt recognition and treatment, if necessary, are important for successful outcome. Treatment options include pericardiocentesis, oxygen therapy for nitrogen wash-out and treating the underlying/associated other air-like syndromes like pneumothorax. We report a case of spontaneous pneumopericardium in a term neonate without any underlying lung pathology or exposure to positive pressure ventilation. The patient was treated successfully with oxygen therapy and discharged home without complications.
\end{abstract}

Volume 5 Issue 2 - 2018

\author{
Amy Haley, Prasanthi Koduru Mishra, Fayez \\ Bany-Mohammedi \\ Department of Pediatrics, University of California at Irvine, USA
}

\begin{abstract}
Correspondence: Fayez Bany-Mohammed, Department of Pediatrics, University of California at Irvine, Division of Neonatal-Perinatal Medicine, California, 101 The City Drive So, Bldg 56, Suite 600, Orange, CA 92868-3298, Tel (7I4) 456-6920, Fax (7।4) 456-7658, Email fbanymoh@uci.edu
\end{abstract}

Received: March 23, 2018 | Published: April 27, 2018

Keywords: newborn, pneumopericardium

\section{Introduction}

Neonatal pneumopericardium is the least common form of airleak syndromes. It can lead to serious complications including sudden cardiac arrest. It usually occurs in association with or is preceded by other air-leak syndromes like pulmonary interstitial emphysema, pneumothorax, pneumomediastinum and subcutaneous emphysema. ${ }^{1}$ Neonatal pneumopericardium has been well described in neonates with respiratory disorders that require assisted ventilation including respiratory distress syndrome, congenital pneumonia and meconium aspiration syndrome. ${ }^{2,3}$ Premature infants with respiratory distress syndrome requiring mechanical ventilation ${ }^{4,5}$ or even nasal continuous positive airway pressure ${ }^{6-8}$ are particularly a higher risk group to develop pneumopericardium. Prompt recognition and treatment are vital for successful outcome. Treatment options include pericardiocentesis with pericardial drainage,$^{5}$ oxygen therapy for nitrogen wash-out ${ }^{9-12}$ and treating the underlying/associated other air-like syndromes like pneumothorax. ${ }^{13}$ Full-term infants with respiratory disorders like meconium aspiration syndrome can develop pneumopericardium $;^{2}$ however, pneumopericardium in fullterm infants with no lung pathology is exceedingly rare. ${ }^{10-13}$ To our knowledge, this report represents the $5^{\text {th }}$ case of such entity ever described in the past 30 years.

\section{Case report}

The patient was delivered at $404 / 7$ weeks gestation via precipitous normal spontaneous vaginal delivery to a 36-year-old Gravida 1, Para 1 mother. He was appropriate for gestational age with a birth weight of $2850 \mathrm{~g}$. Apgar scores were 7 and 8 at 1 and 5minutes respectively. Initial resuscitation included tactile stimulation and bulb suctioning of the nares and oropharynx. Free flow oxygen was given briefly, but bag-mask ventilation was not given in the delivery room. Around 30 minutes of life, the infant developed signs of respiratory distress including grunting, mild subcostal retractions, tachypnea, tachycardia and a briefly documented oxygen desaturation to $80 \%$. He was transferred to the neonatal intensive care unit for further evaluation and monitoring. Initial capillary blood gas showed mild respiratory alkalosis with $\mathrm{pH}$ 7.47, $\mathrm{Pa} \mathrm{CO}, 32 \mathrm{mmHg}, \mathrm{PaO}_{2} 37 \mathrm{mmHg}$ and $\mathrm{HCO}_{3}$ $23 \mathrm{mEq} / \mathrm{L}$. The chest radiograph (CXR) obtained upon admission (30 minutes of life) revealed a pneumopericardium with a classic "halo" sign and a small right-sided pneumothorax (Figure 1). The patient's persistent tachycardia prompted an urgent echocardiogram for evaluation of possible cardiac tamponade. The heart, however, was not well visualized secondary to the presence of air around the heart; otherwise, the study was unremarkable. The patient's clinical status subsequently stabilized with improved tachycardia and tachypnea. There were no other clinical signs to suggest cardiac tamponade like poor perfusion, hypotension or narrow pulse pressure. He was treated with oxygen hood $\left(\mathrm{FiO}_{2} 1.0\right)$ for nitrogen wash-out. A repeat $\mathrm{CXR}$ at 8 hours of life showed persistent but improved pneumopericardium and pneumothorax (Figure 2). The pneumopericardium eventually resolved on subsequent CXR obtained at 15 hours of life (Figure 3) and the oxygen hood was discontinued. The infant remained stable on room air for the remaining duration of his hospital stay. An evaluation for neonatal sepsis, initiated at admission, was negative. He was discharged home after three days of hospitalization without any complications.

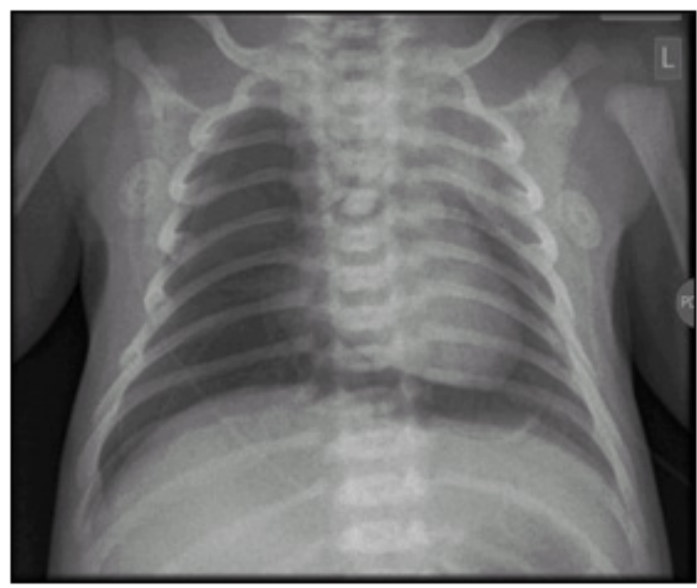

Figure I Large pneumopericardium outlining the cardiac silhouette at 30 minutes of life. 


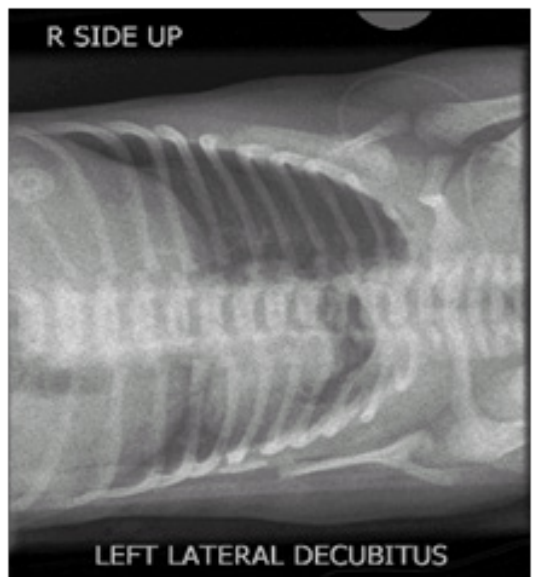

Figure 2 Improved pneumopericardium at 8 hours of life.

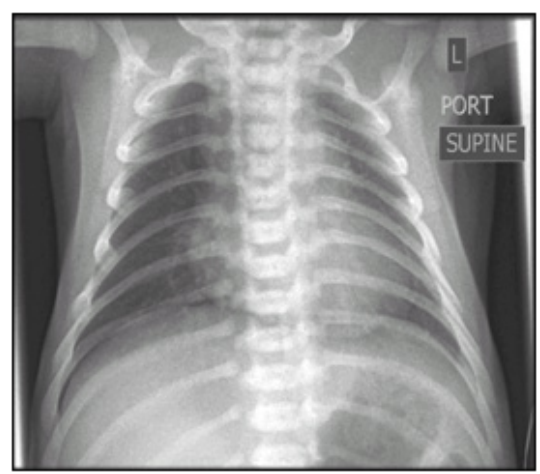

Figure 3 Resolution of pneumopericardium at 15 hours of life.

\section{Discussion}

Pneumopericardium is a rare form of air-leak syndromes that is associated with significant morbidity and mortality. It usually occurs within the first few days of life. ${ }^{3}$ Pneumopericardium generally occurs in association with other air leaks, ${ }^{1,4,5}$ and the incidence is increased in patients with an underlying pulmonary disease such as respiratory distress syndrome, ${ }^{5}$ after vigorous resuscitation, or in the presence of assisted ventilation., ${ }^{3,14,15}$ There have been a handful of reported cases of pneumopericardium that have occurred with the use of continuous positive airway pressure (CPAP).$^{6-8}$ However, only four cases of pneumopericardium occurring in term infants in the absence of resuscitative procedures, parenchymal lung disease or assisted ventilation have been reported ${ }^{10-13}$ in the past 30 years. Other described cases of neonatal pneumopericardium have been associated with infection or trauma. ${ }^{3}$

Mechanical ventilation is a significant risk factor for neonatal pneumopericardium. ${ }^{4}$ High mechanical peak inspiratory pressure (PIP) can cause barotrauma with alveolar over-distention (volutrauma) and potential for rupture. Alveolar over-distension has been reported in patients requiring a high level of positive end-expiratory pressure (PEEP) and subsequently developed pneumopericardium. ${ }^{15}$ The mechanism of pneumopericardium occurring as the result of barotrauma and volutrauma is presumed to be the result of alveolar rupture, which allows air to leak into the interstitial tissue. The air then dissects along the perivascular and peribronchial connective tissue sheaths to the hilum. From there, the air enters the pericardial space at the site of reflection of the parietal pericardium onto the visceral pericardium thereby producing the pneumopericardium. ${ }^{16}$ Moreover, the decreased number and size of Kohn pores in newborns leads to an inability for air to equilibrate between aerated and nonaerated alveoli. ${ }^{8}$ The mechanism of spontaneous pneumopericardium in term infants with no mechanical ventilation or lung pathology is unclear. We presume that the high inflation pressure generated when neonates cry during the first few breaths may occasionally result in an alveolar rupture that eventually leads to pneumopericardium as described above.

The clinical spectrum of pneumopericardium can range from an asymptomatic neonate to one with life-threatening cardiac tamponade. ${ }^{6,7}$ Pneumopericardium results in cardiac tamponade when the intrapericardial pressure exceeds the atrial filling pressure by at least $20-25 \mathrm{~cm} \mathrm{H}_{2} \mathrm{O}$ in a normovolemic subject. This is most likely to happen in patients receiving positive pressure ventilation. ${ }^{16}$ The diagnosis is suggested by symptoms such as hypotension, hypoxemia, bradycardia, and muffled heart sounds. Cardiac tamponade secondary to pneumopericardium must be relieved immediately by simple pericardiocentesis. If recurs, then pericardial tube placement for continuous decompression may be necessary. ${ }^{5,7}$

The classic radiographic finding in pneumopericardium is the "halo" sign, which appears as a continuous radiolucent band of air that outlines the heart and extends to the level of the great vessels. ${ }^{17}$ Pneumopericardium can be distinguished from other air leaks like pleural air and pneumomediastinum by the fact that these may extend above the great vessels and do not collect beneath the heart. An echocardiogram is not necessary or helpful in confirming the diagnosis, but the inability to view the heart through the subxiphoid echocardiographic window can be suggestive of pneumopericardium. ${ }^{18}$ The patient in our case had the classic halo sign on his CXR (Figure 1) which confirmed the diagnosis of pneumopericardium.

In the absence of signs of cardiac tamponade, conservative management may be sufficient with the administration of high oxygen concentrations (nitrogen washout) ${ }^{9-12}$ in the stable full-term infant. However, high oxygen therapy is not advisable in preterm neonates because of the risk of hyperoxia. The patient described in this case was tachycardic but did not exhibit other signs of cardiac tamponade. He was treated with oxygen hood $\left(\mathrm{FiO}_{2} 1.0\right)$ and responded well. In the pre-surfactant era, the mortality rate for very low birth weight infants who developed pneumopericardium associated with mechanical ventilation was reported to be high (up to $83 \%$ ) $^{4,15}$. We believe that both the incidence and mortality from pneumopericardium have decreased after surfactant introduction ${ }^{14}$ however, recent data is lacking. On the other hand, the overall prognosis for spontaneous neonatal pneumopericardium (with or without CPAP) seems to be good..$^{6,8,10-13}$

\section{Conclusion}

This rare case of spontaneous pneumopericardium in a term infant, without underlying lung pathology or assisted ventilation, emphasizes the importance of early clinical detection and the associated excellent prognosis with appropriate management.

\section{Acknowledgements}

None. 


\section{Conflict of interest}

The author declares that there is no conflict of interest.

\section{References}

1. Carey B. Neonatal air leaks: pneumothorax, pneumomediastinum, pulmonary interstitial emphysema, pneumopericardium. Neonatal Netw. 1999;18(8):81-84.

2. Fellous L, Tourneux P, Brulé-Pépin R, et al. Pneumopericardium: a rare complication of meconium aspiration syndrome. Arch Pediatr. 2005;12(1):83.

3. Yeh TF, Vidyasagar D, Pildes RS. Neonatal Pneumopericardium. Pediatrics. 1974;54:429-33.

4. Hook B, Hack M, Morrison S, et al. Pneumopericardium in very low birth weight infants. J Perinatol. 1995;15(1):27-31.

5. Emery RW, Foker J, Thompson TR, et al. Neonatal pneumopericardium: a surgical emergency. Ann Thorac Surg. 1984;37(2):128-132.

6. Türkbay D, Dilmen U, Altug N. Pneumopericardium in a term infant on nasal continuous positive airway pressure. Arch Dis Child Fetal Neonatal Ed. 2007;92:F168.

7. Heckmann M, Lindner W, Pohlandt F. Tension pneumopericardium in a preterm infant without mechanical ventilation: a rare cause of cardiac arrest. Acta Pediatr. 1998;87(3):346-348.

8. Junghaenel S, Sreeram N, Demant A, et al. Pneumopericardium as a rare complication of continuous positive airway pressure in spontaneously breathing neonates. Klin Padiatr. 2012;224(1):34-35.
9. Hummler HD, Bandstra ES, Abdenour GE. Neonatal fellowship. Neonatal pneumopericardium: successful treatment with nitrogen washout technique. J Perinatol. 1996;16(6):490-493.

10. Suresh P, Tagare A, Kadam S, et al. Spontaneous pneumopericardium in a healthy full-term neonate. Indian J Pediatr. 2011;78(11):1410-1411.

11. Björklund L, Lindroth $\mathrm{M}$, Malmgren $\mathrm{N}$, et al. Spontaneous pneumopericardium in an otherwise healthy full-term newborn. Acta Paediatr Scand. 1990;79(2):234-236.

12. Itani MH, Mikati MA. Early onset neonatal spontaneous pneumopericardium. J Med Liban. 1998;46:165-167.

13. Roychoudhury S, Kaur S, Soraisham AS. Neonatal Pneumopericardium in a Nonventilated Term Infant: A Case Report and Review of the Literature. Case Rep Pediatr. 2017;2017:3149370.

14. Walker TL, Shannon DA. Pneumopericardium in the Neonate. Neonatal Netw. 2017;36(6):368-373.

15. Cohen DJ, Baumgart S, Stephenson LW. Pneumopericardium in neonates: is it PEEP or is it PIP? Ann Thorac Surg. 1983;35(2):179183.

16. Trujillo MH, Fragachan CF, Tortoledo F. Cardiac Tamponade due to pneumopericardium. Cardiology. 2006;105(1):34-36.

17. Burt TB, Lester PD. Neonatal pneumopericardium. Radiology. 1982;142:81-84.

18. Allgood NL, Brownlee JR, Green GA. Inability to view the heart through the subxiphoid echocardiographic window: a harbinger of disaster. Pediatr Cardiol. 1994;15(1):27-29. 